\title{
ON THE APPLICATION OF PRINCIPLES OF ARTIFICIAL INTELLIGENCE FOR EIGENSTRAIN RECONSTRUCTION OF VOLUMETRIC RESIDUAL STRESSES IN NON-UNIFORM INCONEL ALLOY 740H WELDMENTS
}

\author{
Fatih Uzun and Alexander M Korsunsky
}

MBLEM, Department of Engineering Science, University of Oxford, Parks Road, Oxford OX1 3PJ, U.K.

fatihuzun@me.com, fatih.uzun@eng.ox.ac.uk

Corresponding author: alexander.korsunsky@eng.ox.ac.uk

\section{GRAPHICAL ABSTRACT}

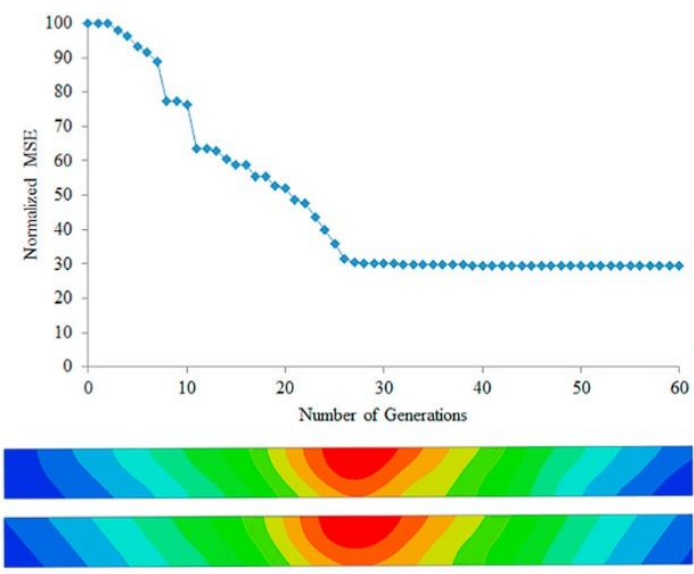

The artificial agent determines the model variables that provide the best match between calculated displacements and experimental measurements.

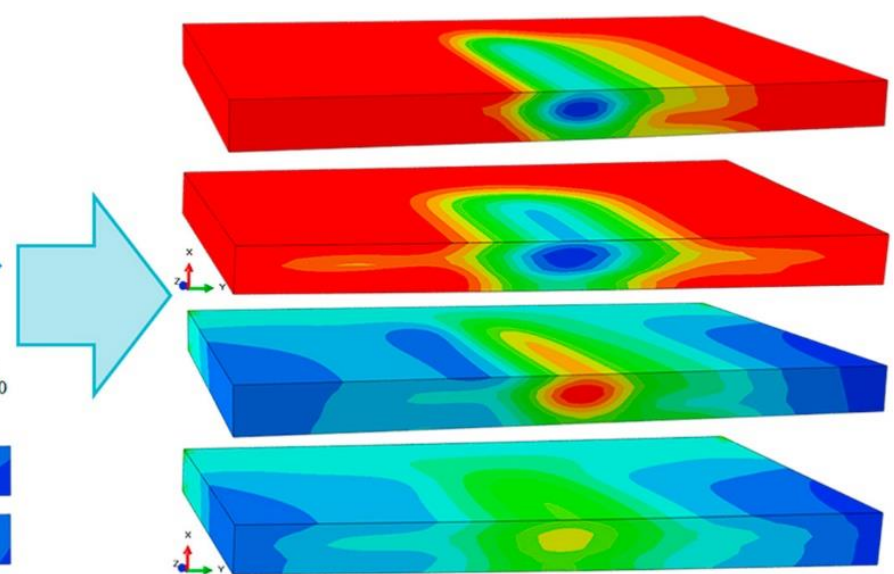

Deterministic finite element model uses the eigenstrain distribution that provides the best displacement match to calculate volumetric residual stress distribution.

Preprint version. Published (recommended) version available in Finite Element in Analysis and Design (2018): https://doi.org/10.1016/j.finel.2018.11.004 


\section{ABSTRACT}

The eigenstrain theory provides a range of fruitful concepts for advanced modelling of the behaviour of materials and components obtained using sophisticated manufacturing routes, their response to thermal and mechanical loading, and deformation under fatigue and creep conditions. In recent years the method has been shown to be able to provide predictions of residual stresses for a limited range of processing and simulated service conditions for which experimental data is available. The authors recently presented advances in the use of eigenstrain-based analysis to include accurate determination of the domain and boundaries of eigenstrain fields in the weld zone. This approach allowed effective modelling of large-scale components and the determination of volumetric distributions of residual stresses through the use of additional model coefficients that need to be determined. Due to the non-linear dependence of the prediction on these parameters, the algorithm of the decision-making process has a profound influence on the cost of the simulation, and the reliability of its output. To address this challenge, the principles of Artificial Intelligence were adopted for use in the eigenstrain contour method to develop fuzzy Finite Element Model (fFEM) for the eigenstrain the reconstruction of residual stresses in large structures. The deterministic finite element eigenstrain model uses contour measurements for reconstruction process, and the developed fFEM behaves as an artificial agent to determine the coefficients of the deterministic finite element eigenstrain model. As an example application, as-welded and post-weld heat-treated specimens of non-uniform weldments of Inconel Alloy $740 \mathrm{H}$ were investigated using the proposed model. The results were verified using displacement measurements and residual stress calculations of the contour method. The determination of model coefficients by artificial agent allowed effective reconstruction of volumetric residual stresses in complex 
shaped components using limited data without the requirement of costly and destructive multi-cut experimental procedures.

Keywords: volumetric residual stress; eigenstrain contour method; artificial intelligence; fuzzy finite element model; evolutionary algorithms

\section{NOMENCLATURE}

$\{A\} \quad$ vector of unknown coefficients of basis functions

$A_{l} \quad$ coefficient of $l^{\text {th }}$ basis function

[C] matrix of calculated displacements

$F_{l} \quad l^{\text {th }}$ basis function

$M_{i} \quad$ mutation search range for $i^{\text {th }}$ variable in vector of model variables

$P_{l, i} \quad$ polynomial function of $l^{\text {th }}$ basis function for short-transverse distribution

$P_{l, j} \quad$ polynomial function of $l^{\text {th }}$ basis function for long-transverse distribution

$\{V\} \quad$ vector of model variables

$V_{i} \quad i^{\text {th }}$ variable in vector $\{V\}$

$X_{p} \quad$ measured displacement at $p^{\text {th }}$ point

$Y_{p} \quad$ calculated displacement at $p^{\text {th }}$ point

$f^{-} \quad$ factor for contraction of $f$

$f^{+} \quad$ factor for expansion of $f$

$\{u\} \quad$ vector of measured displacements

$\varepsilon^{*} \quad$ sum of two components of eigenstrain

$\varepsilon_{y y}^{*} \quad$ long-transverse component of eigenstrain

$\varepsilon_{Z Z}^{*} \quad$ longitudinal component of eigenstrain 
$C x$

Cy

$D x$

Dy

Gx

Gy

MSE

$S a$

$S b$

$d x$

$d y$

$d z$

$f$

$n$

$\alpha$

$\beta$ $\mathrm{x}$-coordinate of centre of eigenstrain field

$y$-coordinate of centre of eigenstrain field

parameter for short-transverse distribution size of Gauss function

parameter for long-transverse distribution size of Gauss function

Gauss function for short-transverse distribution

Gauss function for long-transverse distribution

fitness function in terms of mean squared error

first constant for sigmoidal curve distribution

second constant for sigmoidal curve distribution

short-transverse distance from centre of eigenstrain field

long-transverse distance from centre of eigenstrain field

longitudinal distance from EDM-cut surface

constant for modification of mutation search ranges

total number of points of displacements

coefficient for long-transverse component of eigenstrain

coefficient for longitudinal component of eigenstrain 


\section{INTRODUCTION}

Properties, reliability and operation behaviour of the materials used for steam turbine components are influenced by the welding process. According to the report by Cerjak [1], this process has various applications in steam turbine components. Welding is used for joining and repair purposes, but gives rise to internal residual stresses. Various methods can be used for the measurement of welding residual stresses, such as diffraction [2], contour method [3], and ultrasonic methods [4,5]. It should be noted that diffraction methods are expensive, contour methods are destructive, and ultrasonic methods typically do not offer the necessary resolution and sensitivity.

According to Masubuchi [6], the use of computing methods for the purpose of weld modelling dates back to 1960s. Over the decades, advancements in the computational power allowed the development of numerical models for understanding the mechanism underlying the formation of welding residual stress. Thermal elastic-plastic, inherent strain and inverse eigenstrain methods were developed as the basis for numerical modelling. In the recent years, new computational methods specific to the manufacturing processes were also developed, e.g. Amouzegar et.al. [7] and Fang et. al. [8]. The inverse eigenstrain method combines the benefits of numerical simulations with experimental data obtained using X-Ray and neutron diffraction techniques, or contour measurements. Several examples of this method can be found in the monograph by Korsunsky [9]; the approaches presented allow mapping of residual stress fields based on limited experimental data [10-13]. DeWald and Hill [14] first used contour measurements for the determination of eigenstrain distribution to reconstruct volumetric residual stresses using destructive multi-cut experimental procedures. Uzun and Korsunsky [15] presented improvements in the reconstruction of volumetric residual stress 
fields in complex structures. However, increasing the complexity brings additional model coefficients, and accurate determination of these coefficients requires hundreds of trials and high amount of computation power that limit the practical applicability of the process.

The determination of eigenstrain fields using experimental data eliminates the requirement of complex elastic-plastic calculations for modelling the residual stress evolution in the entire body of a structure, but eigenstrain analysis of non-uniform structures requires careful examination. Uncertainties related to the model should be eliminated by the judicious decisions by the model designer. Time consuming trial and error procedures do not guarantee finding the optimum solution. In order to deal with this problem, smart models that determine the coefficients of a problem without the requirement of intervention of the model designer can offer a solution.

Russel and Noving [16] defined artificial intelligence as the study and design of intelligent agents, where an intelligent agent is a system that perceives its environment and takes actions that maximize its chances of success. In the absence of a smart model, the model designer perceives the information delivered by the model and makes decisions regarding the model setup to maximize success. This study aims to replace the human agent with an artificial agent that searches for model parameters to obtain the solution with maximum success. This process eliminates the requirement of tens of hundreds of manual trials required to make a decision on the model parameters.

Several attempts have been made to combine the finite element method and artificial intelligence-based techniques to model manufacturing processes. Quiza et. al. [17] classified models for combining artificial intelligence with finite element method into four categories 
which are FEM/AI, AI/FEM, hybrid, and fuzzy models. Among these four approaches, fuzzy models deal with uncertainties involved in a model due to the lack of knowledge. They are developed for dealing with coefficients that have unknown probability distributions. In spite of the fact that fuzzy models have an immense potential for dealing with uncertainties faced during the modelling of manufacturing processes, the method has limited number of reported applications in this field. Abdel-Tawab and Noor [18] performed uncertainty analysis of welding residual stresses using a fuzzy logic based finite element model. Principles of fuzzy analysis were integrated with a finite element model for the purpose of structural analysis by Akpan et. al. [19]. Na et. al. [20] used fuzzy neural network models with a finite element analysis procedure to predict residual stresses.

Longitudinal residual stresses in a uniformly welded thin body can be reconstructed by determining only the distribution of a eigenstrain single component, because the magnitude of short and long-transverse residual stresses are much lower than the longitudinal residual stresses. In the case of complex shaped weldments, all components of residual stress can have significant influence on structural integrity. Accurate reconstruction process necessitates the determination of all relevant eigenstrain components. Previous studies [21] showed that transverse residual stresses have high influence in bead-on-plate specimens, and accordingly eigenstrain reconstruction process of this kind of weldments require inclusion of the longtransverse component of eigenstrain. Uzun and Korsunsky [15] previously showed that, in the absence of sufficient data, the reconstruction process for multi-component eigenstrain distribution fail. This solution process necessitates accurate determination of coefficients of eigenstrain components and parameters of functions that are used to define the shape of the eigenstrain distribution, but the non-linear nature of the relation between the model coefficients turns this process into a challenging trial and error task. Accordingly, it can be 
stated that, the inclusion of multiple components of eigenstrain in complex structures creates uncertainties that increase the simulation cost in terms of operator effort and computational power. In this study, the benefits of artificial intelligence are joined with eigenstrain theory in a fuzzy modelling approach for the determination of coefficients of a deterministic finite element eigenstrain model. The proposed model is used for the reconstruction of residual stresses in as-welded and heat-treated non-uniform weldments of Inconel Alloy 740H. The results of example solutions are verified using the experimental data obtained using the contour method.

\section{MATERIALS}

The bead-on-plate weldment design is selected for the development of fFEM because of its complex nature. This design mimics both joining and repair processes on a limited section of a material, and allows the investigation of different stages of the weld process in a single body. As-welded and post-weld heat-treated specimens of Inconel Alloy $740 \mathrm{H}$ were prepared according to the dimensions given in Figure 1. The centre of the Cartesian coordinate system is placed at the centre of the yz plane at the bottom of the plate. The thickness of plate was 12 $\mathrm{mm}$, and its length and width were 200 and $150 \mathrm{~mm}$, respectively. The weld slots had approximately $12 \mathrm{~mm}$ width and $67 \mathrm{~mm}$ length at the top surface and $8 \mathrm{~mm}$ width and $63 \mathrm{~mm}$ length at their bottom section. The top length of the weld bead was set to cover $1 / 3$ of the plate length. The nominal chemical composition of the alloy was chromium, $24.5 \%$, cobalt, $20.0 \%$, aluminium, $1.35 \%$, titanium, $1.35 \%$, and niobium, $1.5 \%$, nickel balance. Iron, carbon, manganese, molybdenum, silicon, copper, phosphorus, sulphur and boron elements were included at nominal ratios. Subsequent to the machining, specimens were pre-weld annealed at $1107^{\circ} \mathrm{C}$ for 1 hour and water cooled. Weld slots were filled with three weld beads 
using tungsten inert gas (TIG) welding technique. Parameters of the welding process are given in Table 1. The filler material had the same composition as the base plate. One of the specimens was post-weld heat treated (aged) at $800^{\circ} \mathrm{C}$ for 4 hours and air cooled.

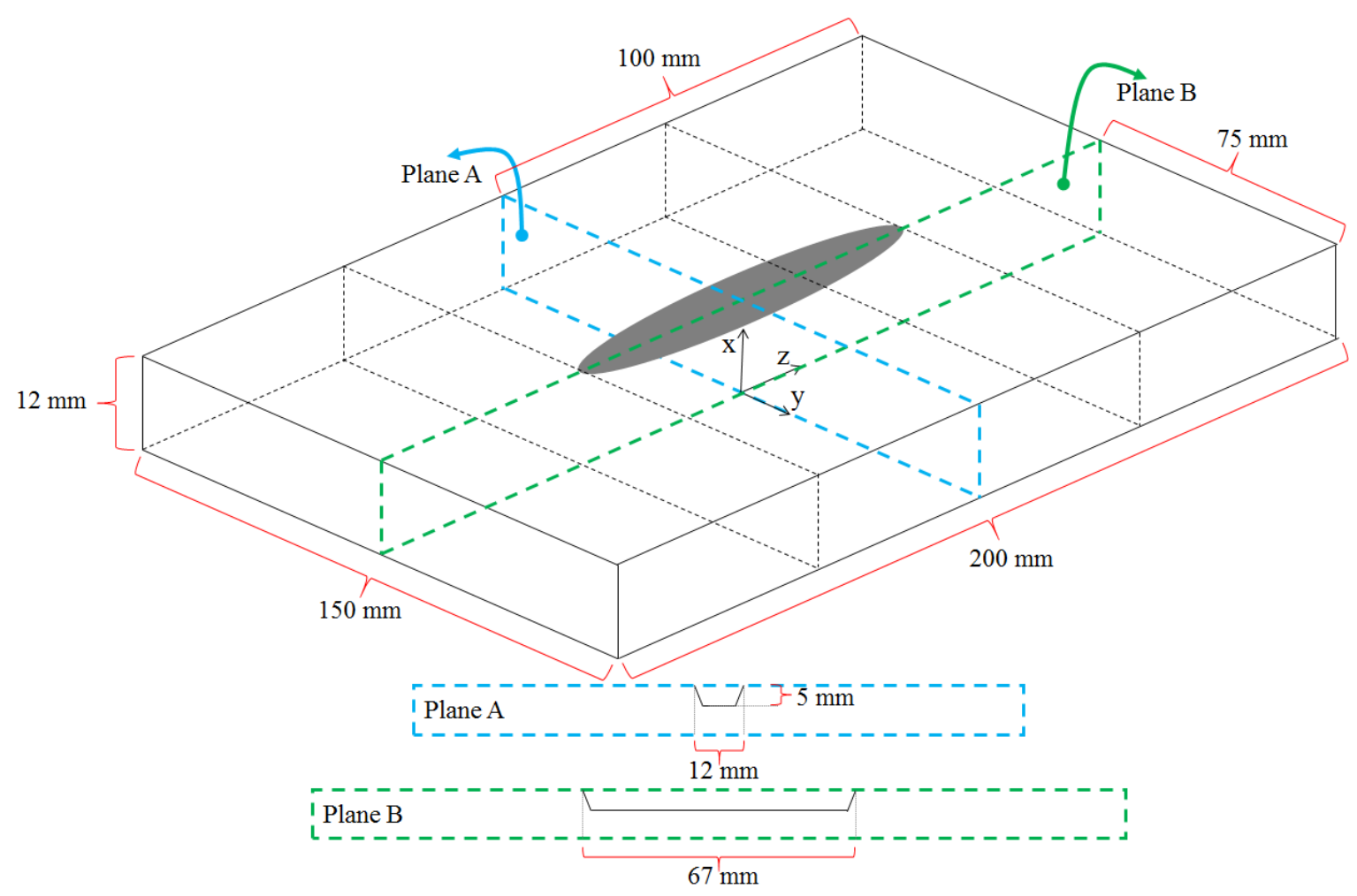

Figure 1. 3D representation of the model geometry of Inconel Alloy $740 \mathrm{H}$ weldments

The eigenstrain contour method for the reconstruction of residual stresses is based on matching the displacements following the stress relief obtained by contour measurements from a cut surface created using electric discharge machining (EDM) technique. In this study, both specimens were cut at Plane A for contour measurements. The elastic modulus was set to $221 \mathrm{GPa}$ for linear elastic calculations of deterministic finite element eigenstrain model. Detailed material properties of Inconel Alloy $740 \mathrm{H}$ can be found in the data sheet provided by Special Metals Corporation [22]. 
Table 1. Parameters for TIG welding of as-welded (AW) and post-weld heat-treated (PWHT) specimens of Inconel Alloy $740 \mathrm{H}$

\begin{tabular}{lcc|cc|cc} 
& \multicolumn{2}{c}{ Weld Pass 1 } & \multicolumn{2}{c}{ Weld Pass 2 } & \multicolumn{2}{c}{ Weld Pass 3 } \\
& AW & PWHT & AW & PWHT & AW & PWHT \\
\cline { 2 - 7 } & 174.0 & 174.0 & 174.0 & 174.0 & 174.0 & 174.0 \\
Current (A) & 16.1 & 16.0 & 16.3 & 16.5 & 16.3 & 16.2 \\
Voltage (V) & 163.0 & 136.4 & 152.4 & 111.5 & 134.3 & 123.4 \\
Travel Speed (mm/min) & 177.8 & 254.0 & 228.6 & 304.8 & 177.8 & 228.6 \\
Wire Speed (mm/min) & & & & & & \\
\hline
\end{tabular}

\section{THE FUZZY MODEL}

Residual stresses are the stresses that are accommodated in a material without any external load. Understanding their distribution in a material has vital importance for structural integrity, but the determination of these stresses is a challenging task. Ahn et al. [23] state that it is impossible to get a full map of six components of residual stresses in engineering components using currently available expensive and time-consuming methods. Therefore, thermal-structural elastic-plastic finite element models of residual stress evolution are widely used, but these models require detailed knowledge of heat source geometry, temperaturedependent material properties, and precise dimensions of the material. The high number of unknowns reduces the credibility of these models because of high deviations of results from experimental measurements. In order to deal with these difficulties, Mura [24] introduced the eigenstrain reconstruction method that combines the accuracy of high density experimental data (e.g. from contour measurements) with elastic finite element models. The idea behind this approach to use the main source of residual stresses, eigenstrains, for the calculation of residual stresses in numerical models with a low number of degrees of freedom. 
Combining experimental data with linear elastic finite element models allows reliable prediction of residual stresses, but complexities of a structure create uncertainties in this kind of models [15]. In order to deal with this problem, principles of artificial intelligence are used for the development of a smart model, fuzzy finite element model (fFEM), that determines the coefficients of the deterministic finite element eigenstrain model based on the quantified quality of experimental validation.

According to the principle of superposition, residual stresses relax elastically, and they can be correlated with deformations formed as a result of stress relaxation. The contour method uses this principle to calculate residual stresses using deformations on a planer cut appeared after EDM sectioning process. Reliable results of this method are limited to the stresses normal to the plane of cut. Details of the method can be found in the study of present authors [15]. The same procedure of contour method which is explained in that study is used for experimental validation of fFEM residual stress calculations.

\subsection{Eigenstrain Contour Method}

Eigenstrain reconstruction process is based on the determination of eigenstrain fields in a body using a series of functions and calculation of the residual stresses. The principles of this well-known process can found in the studies of DeWald and Hill [14] and Kartal et. al. [3]. Previously, it was shown that the main source of welding residual stress is the eigenstrains in and around the weld beam [15]. Authors analysed three different models for solving the inverse eigenstrain problem. Investigations showed that determination of eigenstrain distribution based on a simplifying assumption, that long-transverse and longitudinal components of eigenstrain are identical with a constant ratio, is the most effective method for 
reconstruction of residual stresses. This method maps the distribution of two identical components of eigenstrain within the body using three different functions. For this purpose, a series of Chebyshev polynomials up to $4^{\text {th }}$ order is combined with Gauss function as illustrated in Figure 2 to determine the planar distribution of eigenstrains. In the case of nonlinear bead-on-plate weldment, the variation of the planar eigenstrain distribution along the longitudinal direction is specified using a sigmoidal curve of Knee function which was found by Korsunsky [25].
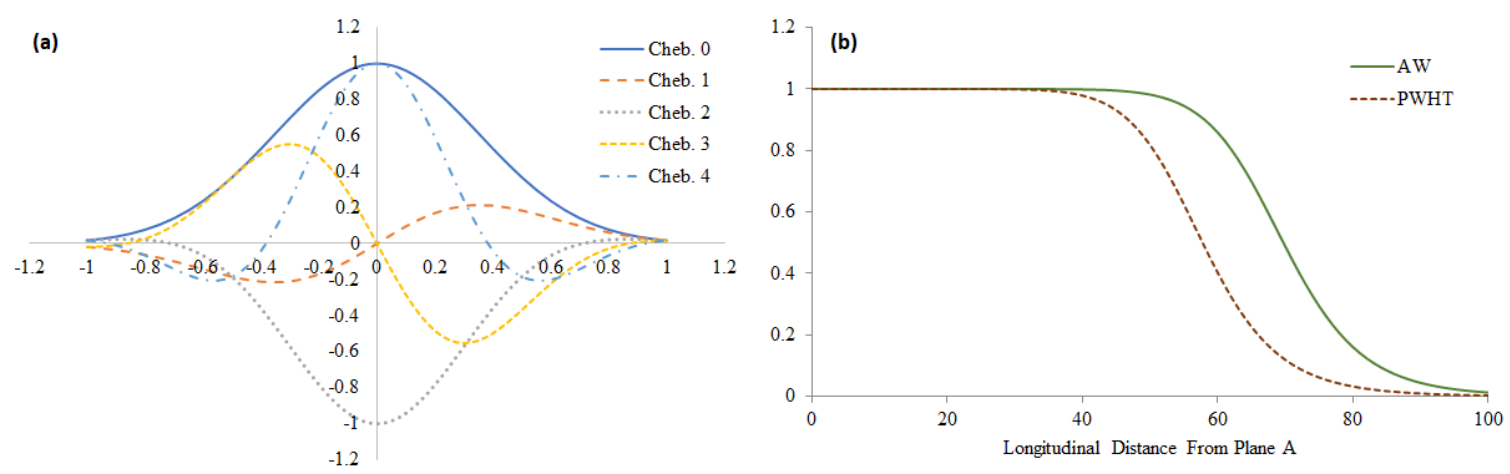

Figure 2. (a) Chebyshev functions up to $4^{\text {th }}$ order combined with Gauss function and (b) final form of Knee function determined by the fFEM in as-welded (AW) and post-weld heattreated (PWHT) conditions

The longitudinal component of eigenstrain $\left(\varepsilon_{z z}^{*}\right)$ is the primary driving force on the formation of welding residual stress in uniformly distributed thin weldments. However, non-uniform weldments require careful investigation of all components of eigenstrain for understanding the role of eigenstrain on formation of welding residual stress. Based on the assumption that short-transverse component of eigenstrain has a minor effect on the formation of welding residual stress, long-transverse $\left(\varepsilon_{y y}^{*}\right)$ and longitudinal $\left(\varepsilon_{z z}^{*}\right)$ components of eigenstrain are included in the reconstruction process of residual stresses and they are assumed to be proportional with fixed coefficients. This approach keeps both eigenstrain terms equal $\left(\varepsilon_{y y}^{*}=\right.$ 
$\varepsilon_{z z}^{*}$ ) but their magnitude are determined using coefficients of eigenstrain which are $\alpha$ and $\beta$ for long-transverse $\left(\varepsilon_{y y}^{*}\right)$ and longitudinal $\left(\varepsilon_{z z}^{*}\right)$ components of eigenstrain respectively. The sum of two components of eigenstrain $\left(\varepsilon^{*}\right)$ is calculated using Equation 1.

$\varepsilon^{*}=\alpha\left[\begin{array}{ccc}0 & 0 & 0 \\ 0 & \varepsilon_{y y}^{*} & 0 \\ 0 & 0 & 0\end{array}\right]+\beta\left[\begin{array}{ccc}0 & 0 & 0 \\ 0 & 0 & 0 \\ 0 & 0 & \varepsilon_{z z}^{*}\end{array}\right]$

Separate components of eigenstrain are formulated in Equation 2 as the sum of linear combinations of unknown polynomial series with the same coefficients $\left(A_{l}\right)$ which are multiplied by the coefficients of eigenstrain $\left(\alpha\right.$ and $\beta$ ). In this equation, $A_{l}$ is the unknown coefficient of $l^{\text {th }}$ term of a series expansion of known basis function $\left(F_{l}\right)$.

$\varepsilon^{*}(x, y)=\alpha\left(\sum_{l=1}^{(m+1)(n+1)} A_{l} F_{l}(x, y)\right)+\beta\left(\sum_{l=1}^{(m+1)(n+1)} A_{l} F_{l}(x, y)\right)$

Planar distribution of eigenstrains is satisfied by combining the polynomial terms for shorttransverse and long-transverse directions. The $l^{\text {th }}$ basis function is determined using Equation 3 where $P_{l, i}$ and $P_{l, j}$ represent $i^{\text {th }}$ and $j^{\text {th }}$ order Chebyshev polynomials combined with Gauss function.

$F_{l}(x, y)=P_{l, i}(x) P_{l, j}(y)$

After calculation of displacements using eigenstrain fields determined by each basis function separately, the unknown coefficients, $\{A\}$, are calculated using the column vector of 
experimentally determined displacement data, $\{u\}$. The solution of this elastic problem is formulated in Equation 4.

$\{u\}=[C]\{A\}$

In Equation 4, the column vector $\{A\}$ contains coefficients of $w$ number of basis functions as it is given in Equation 5:

$\{A\}=\left[A_{1}, A_{2}, \ldots, A_{w}\right]^{T}$

The model derived matrix, $[C]$, in Equation 4 is composed of displacements obtained by solving basis functions. The matrix has $w$ number of columns for each basis function and $r$ number of rows corresponding to each individual data point as it is given in Equation 6.

$[C]=\left[\begin{array}{ccc}C_{1,1} & \cdots & C_{w, 1} \\ \vdots & \ddots & \vdots \\ C_{1, r} & \cdots & C_{w, r}\end{array}\right]$

\subsection{The Fuzzy Finite Element Model (fFEM)}

The core deterministic eigenstrain model calculates the coefficients of basis functions by solving the inverse eigenstrain problem to determine residual stresses and displacements but it contains eight different coefficients which are eigenstrain distribution boundaries in shorttransverse $(D x)$ and long-transverse directions $(D y)$, coordinates of the centre of planar eigenstrain distribution $(C x, C y)$, constants that determine the shape of sigmoidal curve 
distribution $(S a, S b)$ and coefficients for eigenstrain components $(\alpha, \beta)$. The vector of model coefficients is given in Equation 7.

$\{V\}=[D x, D y, C x, C y, S a, S b, \alpha, \beta]$

The proposed fFEM aims to determine coefficients of the deterministic finite element eigenstrain model. It is based on an evolutionary process that minimizes the mean squared error $(M S E)$ between displacements calculated by the deterministic finite element eigenstrain model and the contour method measurements. Starting from roughly estimated model coefficients, evolutionary process determines the best possible combination of coefficients. This intelligent process uses genetic algorithms. Equation 8 is used for the calculation of $M S E$ where $Y_{p}$ is the calculated displacement and $X_{p}$ is measured and averaged experimental displacement at point $p$. This is also the fitness function of the evolution process.

$$
M S E=\frac{1}{n} \sum_{p=1}^{n}\left(Y_{p}^{2}(x, y)-X_{p}^{2}(x, y)\right)
$$

The shape of the sigmoidal curve is determined using a modified form of Knee function which is given in Equation 9 where $d z$ represents the distance from the EDM-cut surface. $S a$ and $S b$ are the parameters that define the length and slope of the sigmoidal curve respectively. Gauss functions $(G x, G y)$ that determine eigenstrain distributions in short-transverse and long-transverse directions are given in Equations 10a and 10b respectively where $d x$ and $d y$ represent axial distances from the centre of eigenstrain distribution which is located at the coordinates determined by $C x$ and $C y$. Distance and coordinates used in this function are normalized to 1.0 using the eigenstrain distribution boundaries, $D x$ and $D y$. 
$S=d z^{S b} /\left(S a^{S b}+d z^{S b}\right)$

$$
\begin{aligned}
& G x=\exp \left(-\left(d x / D x-(C x / D x)^{2}\right) / 0.5^{2}\right) \\
& G y=\exp \left(-\left(d y / D y-(C y / D y)^{2}\right) / 0.5^{2}\right)
\end{aligned}
$$

Evolution process starts with the creation of the base population randomly in a search space around the initially estimated coefficients. After the creation of the base population, separate solutions of deterministic core finite element eigenstrain model are performed for each member and fitness values corresponding to these solutions are calculated. Related to the fitness value of each member, base population is evaluated using basic operators of the genetic algorithm, which are selection, crossover, mutation and elitism, and a new population, which is composed of elite members of the previous population and new members, is created. The deterministic finite element eigenstrain model is solved again using the new population and fitness of the best member of the new population is compared with that of the base population. According to this comparison, mutation search range is expanded or reduced. Mutation search range of coefficients in the vector, $\{V\}$, that define each member in the population is determined to be flexible in order to increase the possibility of finding the global minimum. Each gene (each coefficient of the deterministic finite element model) in a chromosome mutated at different ranges related to their scalar value. The mutation range is formulated in Equation 11 where $M_{i}$ is the mutation search range of $i^{\text {th }}$ coefficient in the vector $\{V\}$, and $f$ is a variable that is modified at each generation according to the improvements obtained during the evolution process. If the best member of the population is better than the best member of the previous population, the magnitude of this constant is increased and if MSE is not reduced in the previous generation, its magnitude is decreased by multiplying with factors $\left(f^{+}\right)$and $\left(f^{-}\right)$respectively. The factors used for modification of the 
variable are kept constant in all generations of the evolution process. A similar approach for increasing the possibility of finding the global minimum was previously used by Toklu and Uzun [26], Uzun [27] and Uzun [28] and effective results are obtained during the energy minimization of tensegrity structures. Unless the stop criterion is satisfied, the new population is attained as the base population and a new population is created. The unit-less variable, $f$, is initially determined to be 0.1 . The factors, $f^{+}$and $f^{-}$, for adjusting the strength of this variable are kept as 1.1 and 0.9 respectively.

$M_{i}=V_{i} f$

The flowchart of the fFEM that includes the deterministic finite element eigenstrain model at the core is illustrated in Figure 3. This flowchart summarizes the processes explained in Section 3. fFEM is developed as a Python script for ABAQUS 6.14-5 and solutions are performed using a computer with Intel ${ }^{\circledR}$ Core $^{\mathrm{TM}} \mathrm{i} 7$ processor that has 4 physical cores. Mesh density and number of integration points have a high influence on the reliability of the results and the solution time. Therefore, optimum mesh density should be reached using relevant element type. The cuboid model geometry is meshed by C3D8R general purpose brick element which has reduced integration point. Investigations showed that increasing the number of elements beyond 13720 elements does not provide any improvement in results of the deterministic finite element eigenstrain model, but increases the computation cost exponentially. Therefore, solutions of both fFEM for eigenstrain reconstruction process and contour method for experimental verification of the fFEM are performed using the same model geometry and mesh. 


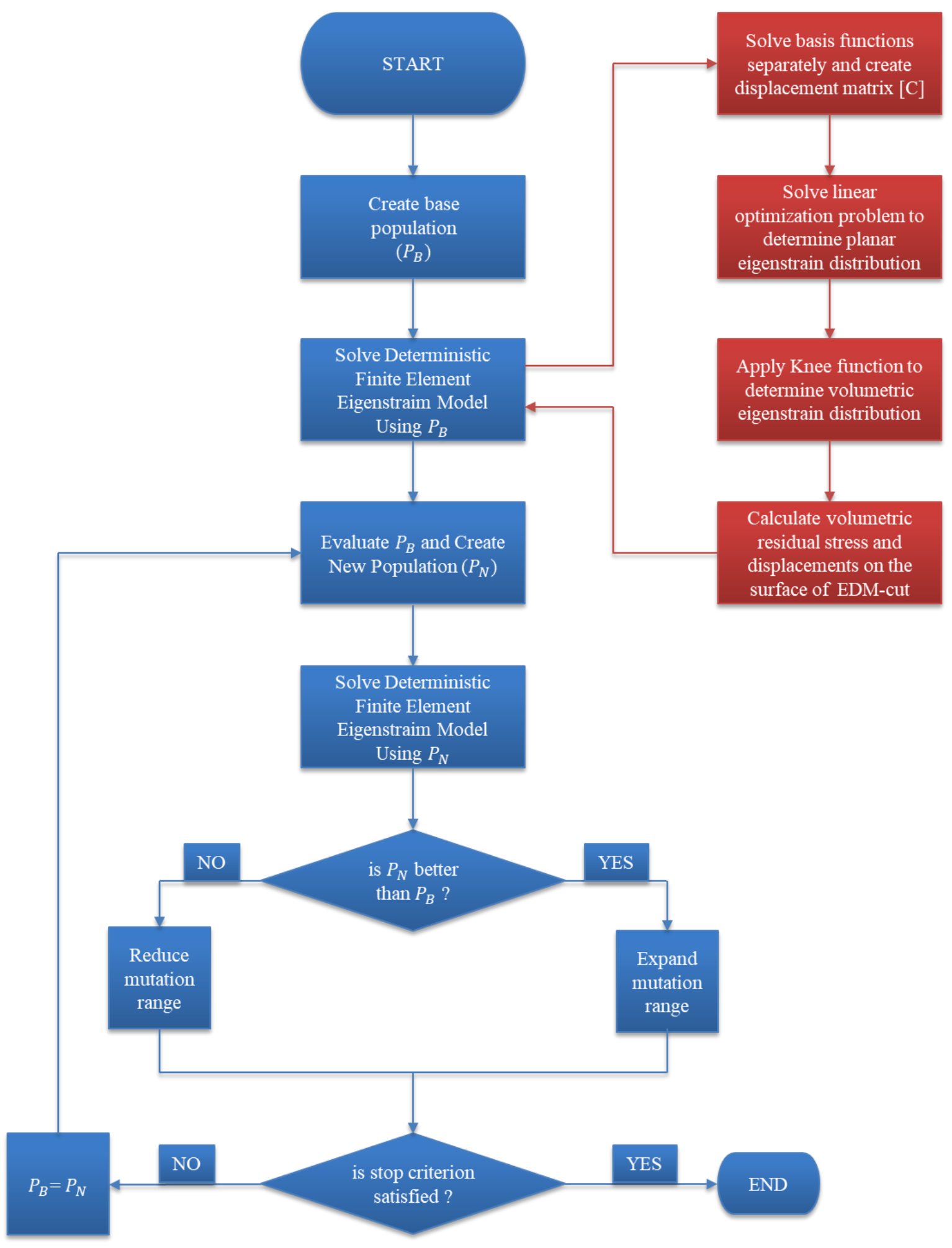

Figure 3. Flow chart of the fFEM (blue) and deterministic finite element eigenstrain model (red) 


\section{RESULTS}

Evolution of coefficients of the deterministic core eigenstrain model is performed by an evolutionary algorithm. Parameters specific to this algorithm are determined to the same for the solutions of as-welded and post-weld heat-treated conditions. Population size is determined to be 5 and 1 member is kept as the elite member which is transferred to the next population after the application of selection, cross-over and mutation operators. Fitness calculations are done after the solution of the deterministic finite element eigenstrain model for each member. Members are selected according to their fitness using pie graph method [29] and single point cross-over is performed with a probability of 0.5 . New members, which are created through the cross-over process, are mutated in a search range which is determined specific to each model coefficient. Mutations in this process are adjusted to take place with a probability of 0.95 .

Model coefficients are evolved at each generation starting from a roughly estimated initial set of coefficients to the final set of coefficients as illustrated in Figure 4. Solutions of as-welded and post-weld heat-treated conditions are started using the same initial set of coefficients and evolved until the optimum set of coefficients are obtained. Evolution of the model coefficients continued for 60 generations. Results show that after 30 generations, both models reached a steady state and improvement in terms of $M S E$ is reduced to insignificant values. Both initial and final sets of coefficients are given in Table 2. 

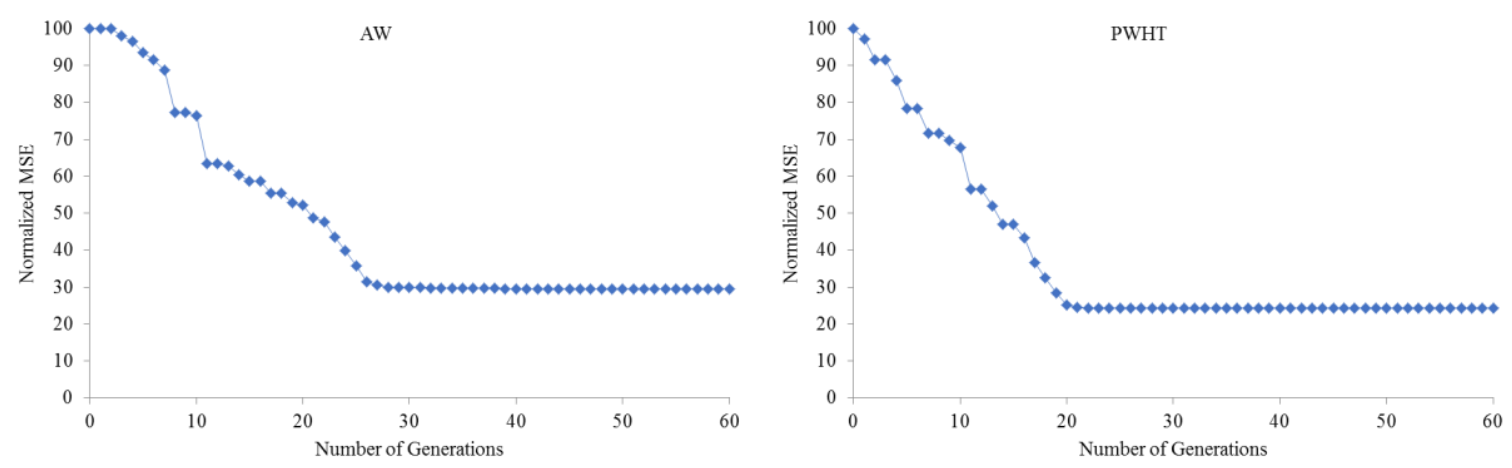

Figure 4. Evolution of the model coefficients during the 60 generations in terms of $M S E$ normalized to 100 in as-welded (AW) and post-weld heat-treated (PWHT) conditions

Model coefficients can be grouped into four categories. Coefficients that determine the distribution size of eigenstrains show that the eigenstrain distribution length in long-transverse direction, $D y$, of the post-weld heat-treated condition is $50 \%$ higher than the as-welded condition. $D x$ values show that eigenstrains are extended through the short-transverse direction in both conditions, but the size of Gauss function in this direction served only as a fine-tuning tool that modifies the quality of fit with experimental data. The second group of coefficients, $C x$ and $C y$, shows that the centre of eigenstrain fields of as-welded and postweld heat-treated conditions seem to be moved in the positive direction of $\mathrm{x}$ and $\mathrm{y}$-axes relative to the geometric centre of Plane A. According to the optimum coefficients of Knee function, the longitudinal length of eigenstrain field, which is determined by $S a$, is longer in the as-welded condition. The coefficient that determines the slope of Knee function, $S b$, shows that as-welded and post-weld heat-treated conditions have similar eigenstrain distribution slopes in the longitudinal direction as illustrated in Figure 2. Final group of model coefficients are the coefficients of eigenstrain, $\alpha$ and $\beta$, which are used to calculate magnitude of separate eigenstrain components. Results show that the influence of long-transverse component of eigenstrain decreases after the heat treatment process. 
Table 2. Optimum coefficients of the deterministic finite element eigenstrain model for aswelded (AW) and post-weld heat-treated (PWHT) conditions

\begin{tabular}{rcccccccc} 
& $\begin{array}{c}D x \\
(\mathrm{~mm})\end{array}$ & $\begin{array}{c}D y \\
(\mathrm{~mm})\end{array}$ & $\begin{array}{c}C x \\
(\mathrm{~mm})\end{array}$ & $\begin{array}{c}C y \\
(\mathrm{~mm})\end{array}$ & $\begin{array}{c}S a \\
(\mathrm{~mm})\end{array}$ & $S b$ & $\alpha$ & $\beta$ \\
\cline { 2 - 9 } Initial & 5 & 10 & 0.005 & 0.01 & 50 & 10 & 1 & 1 \\
AW & 6.791947 & 38.20917 & 0.01015 & 0.06167 & 69.70885 & 12.01218 & 3.012535 & 0.903452 \\
PWHT & 4.892711 & 57.10497 & 0.00903 & 0.05670 & 57.75904 & 10.41161 & 1.270968 & 0.339531 \\
\hline
\end{tabular}

Optimum coefficient sets are used for the determination of volumetric eigenstrain distribution in the whole body of as-welded and post-weld heat-treated specimens, the calculation of displacements on the surface of EDM-cut and the mapping of volumetric residual stress. Results show that longitudinal displacements on the surface of EDM-cut measured using the contour method and calculated using the fFEM are in a complete agreement as illustrated in Figure 5. In order to have a better understanding on the validity of the results, profile plots of through-thickness averages of distributions of displacements and longitudinal residual stresses along the long-transverse direction are compared. Results in Figure 6 show that the displacement and residual stress profiles of the fFEM are in good agreement with experimental results.

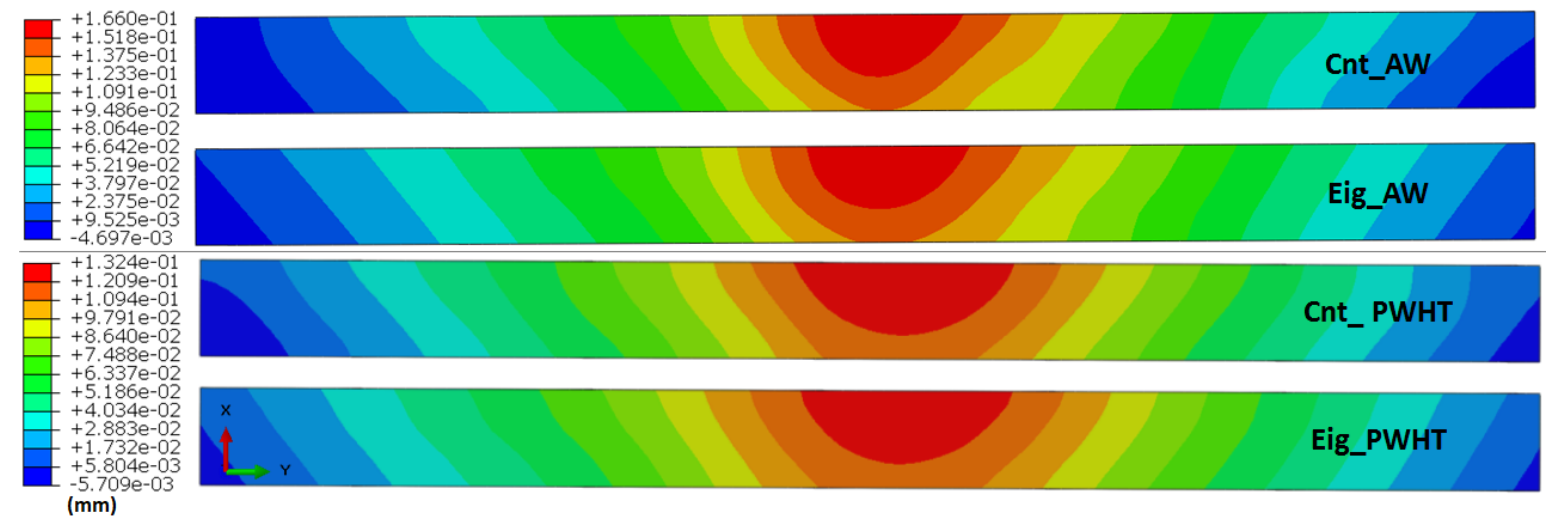

Figure 5. Planar distribution of longitudinal displacements on Plane-A measured using contour method (Cnt) and calculated by the fFEM method (Eig) in as-welded (AW) and postweld heat-treated (PWHT) conditions 

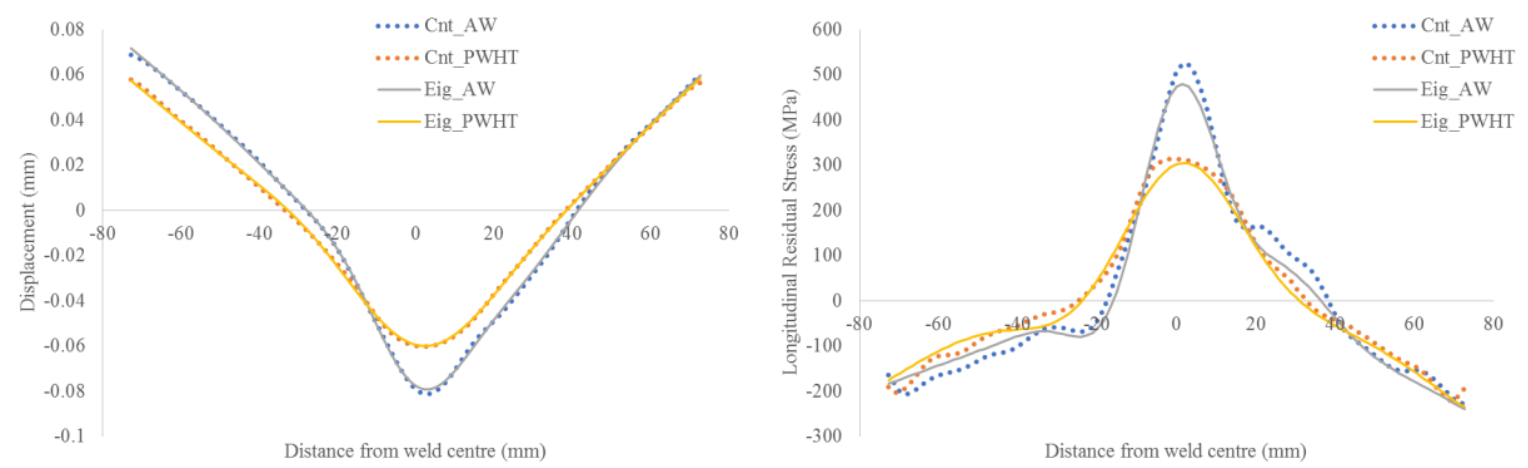

Figure 6. Profile distributions of through-thickness averages of displacements (left) and residual stresses (right) determined using the contour method (Cnt) measurements and the fFEM method (Eig) calculations in as-welded (AW) and post-weld heat-treated (PWHT) conditions

Volumetric distributions of longitudinal residual stresses in the whole body of as-welded and post-weld heat-treated conditions are illustrated in Figure 7. As it is expected, the magnitude of longitudinal tensional residual stresses in the weld zone is lower in the post-weld heattreated condition than it is in the as-welded condition. However, the influence of post-weld heat-treatment process on longitudinal compressive stresses seems to be lower. In order to understand the reason of this, it is necessary to analyse the distribution of eigenstrains in the whole body.

Volumetric distributions of eigenstrains in as-welded and post-weld heat-treated conditions are illustrated in Figure 8, but the scales only represent the field distribution. Magnitudes of $\varepsilon_{y y}^{*}$ and $\varepsilon_{z z}^{*}$ components are calculated after multiplying the field values by coefficients of eigenstrain, which are $\alpha$ and $\beta$. It is observed that the length of eigenstrain field is shortened after the post-weld heat-treatment process but, post-weld heat-treatment is a complex process that causes conversion of elastic strains into creep strain. It can be stated that post-weld heattreatment process causes reduction of eigenstrains, but formation of creep strains increases 
eigenstrains in the regions where residual stress magnitude is relatively high. The expansion observed in the eigenstrain distribution in the long-transverse direction is a result of this mechanism.

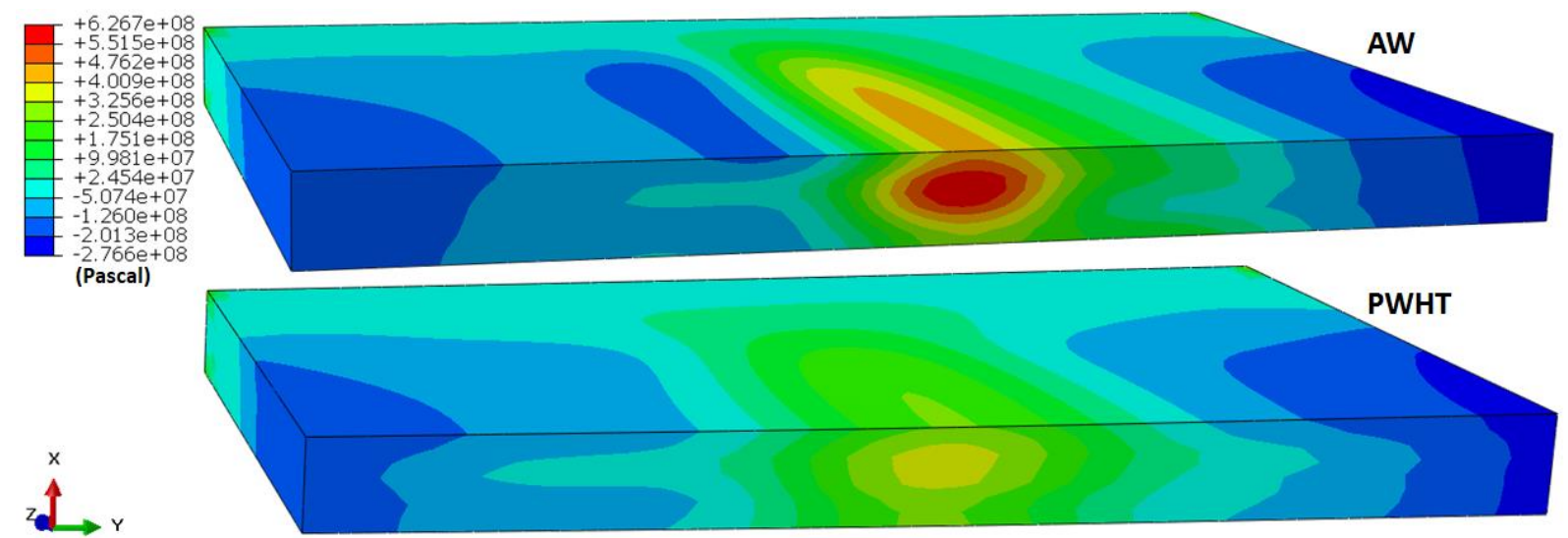

Figure 7. Volumetric distribution of longitudinal residual stresses in as-welded (AW) and post-weld heat-treated (PWHT) conditions calculated using fFEM

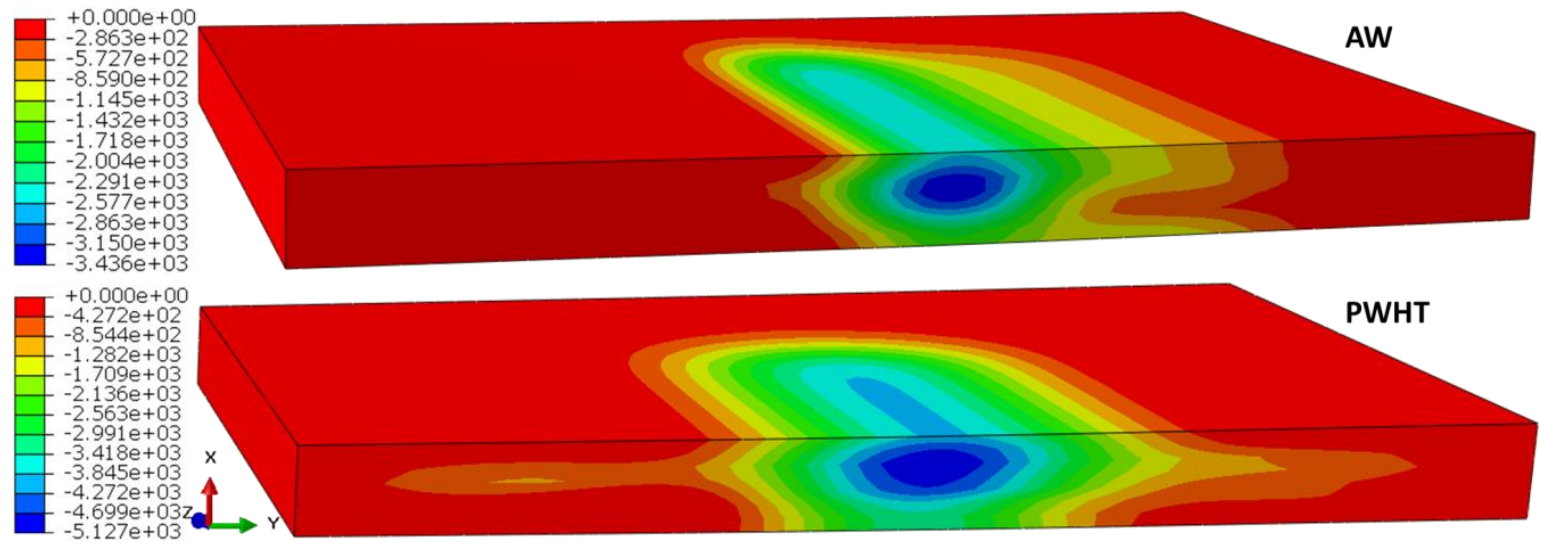

Figure 8. Volumetric distribution of eigenstrains in as-welded (AW) and post-weld heattreated (PWHT) conditions

Considering the material properties presented by the producer of Inconel Alloy $740 \mathrm{H}$ [22], the yield strength of the plates at room temperature is around $740 \mathrm{MPa}$. According to the study of Conduit et. al. [30], the uncertainty of yield strength of nickel-based superalloys can reach up 
to $30 \mathrm{MPa}$. The maximum magnitude of equivalent tensile stress calculated by fFEM in the AW condition is $765 \mathrm{MPa}$. The von-Mises criterion states that a metal starts to yield when equivalent tensile stress is reached to yield strength. In this case, yield strength is exceeded by $25 \mathrm{MPa}$ which is $3.37 \%$ of the yield strength determined by the producer. This small difference is within the limits of uncertainty determined for nickel-based superalloys [30] and, accordingly, it can be stated that maximum equivalent tensile stress is around the yield strength. In addition, investigations on the effect of welding process on hardness showed that welding process causes hardening in the weld zone $[31,32]$ and, therefore, it is possible to have high maximum equivalent tensile stress at the level of yield strength or above in the weld zone.

The nature of eigenstrain contour method provides results that validate themselves by experimental displacement measurements. In addition to the displacement results, residual stress calculations of the contour method are also included in this study to show the high reliability of the proposed fFEM. Further comparisons are done with the studies that use beadon-plate weldment design. In the study of Smith and Smith [33] all non-destructive measurements, which were done using NeT single weld bead-on-plate specimen, are given. Neutron diffraction results for longitudinal residual stress, which distribute through the thickness of the specimen at the centre of weld zone, shows a similar pattern with the results of fFEM. The other result specific to the bead-on-plate specimen design is the formation of compressive longitudinal stresses at the edge parts of the long-transverse direction. This behaviour of residual stress distribution appears because the length of un-welded sections cover $2 / 3$ of the full length of the plate. Similar distribution of longitudinal stresses can be found in the results of measurements and simulations of different laboratories presented by Simith and Simith [33]. 


\section{CONCLUSION}

The study on the identification of eigenstrain sources of welding residual stress [15] previously showed that the main source of welding residual stress is the eigenstrains formed in and around the weld zone. That study allowed mapping eigenstrains within a welded body, but the proposed solution procedure required hundreds of different trials to find a reasonable solution. Using the idea behind the eigenstrain reconstruction process, that is obtaining the best match with experimental measurements, a new algorithm that combines the principles of artificial intelligence with the eigenstrain theory is developed. The proposed fFEM takes decision-making process on the coefficients of the deterministic finite element model of the eigenstrain contour method from the model designer and finds the model coefficients that provide the optimum solution in terms of quantified quality of match between calculated displacements and experimental measurements. The artificial agent improved the reliability of the results and automatized full scale eigenstrain reconstruction process of large components.

\section{ACKNOWLEDGEMENTS}

The Scientific and Technological Research Council of Turkey (TÜBITAK) supported this study through grant Number: TUBITAK BIDEB-2219. European Commission Research Executive Agency (REA) supported this research through grant MSCA-IF no. 794957 (RESTREIG). The authors would like to thank Dr Steve McCoy of Special Metals Corporation for providing Inconel Alloy $740 \mathrm{H}$ specimens. 


\section{REFERENCES}

[1] H.H. Cerjak, Welding of steam turbine components, Study report of the COST 505 Welding Group, 1992.

[2] M.E. Fitzpatrick, A. Lodini, Analysis of Residual Stress by Diffraction Using Neutron and Synchrotron Radiation, 2003. doi:10.1088/0957-0233/14/9/703.

[3] M.E. Kartal, Y.H. Kang, A.M. Korsunsky, A.C.F.F. Cocks, J.P. Bouchard, The influence of welding procedure and plate geometry on residual stresses in thick components, Int. J. Solids Struct. 80 (2016) 420-429. doi:10.1016/j.ijsolstr.2015.10.001.

[4] P. Palanichamy, M. Vasudevan, T. Jayakumar, Measurement of residual stresses in austenitic stainless steel weld joints using ultrasonic technique, Sci. Technol. Weld. Join. 14 (2009) 166-171. doi:10.1179/136217108X394753.

[5] F. Uzun, A.N.A.N. Bilge, Ultrasonic Investigation of the Effect of Carbon Content in Carbon Steels on Bulk Residual Stress, J. Nondestruct. Eval. 34 (2015). doi:10.1007/s10921-015-0284-X.

[6] K. Masubuchi, Models of Stresses and Deformation Due to Welding-A Review, JOM J. Miner. Met. Mater. Soc. 33 (1981) 19-23. doi:10.1007/BF03339550.

[7] H. Amouzegar, B.W. Schafer, M. Tootkaboni, An incremental numerical method for calculation of residual stresses and strains in cold-formed steel members, Thin-Walled Struct. 106 (2016) 61-74. doi:10.1016/j.tws.2016.03.019.

[8] H. Fang, T.M. Chan, B. Young, Material properties and residual stresses of octagonal high strength steel hollow sections, J. Constr. Steel Res. 148 (2018) 479-490. doi:10.1016/j.jcsr.2018.06.007.

[9] A.M. Korsunsky, A Teaching Essay on Residual Stresses and Eigenstrains, 
Butterworth-Heinemann, Oxford, United Kingdom, 2017.

[10] E. Salvati, T. Sui, A.J.G. Lunt, A.M. Korsunsky, The effect of eigenstrain induced by ion beam damage on the apparent strain relief in FIB-DIC residual stress evaluation, Mater. Des. 92 (2016) 649-658. doi:10.1016/j.matdes.2015.12.015.

[11] E. Salvati, A.M. Korsunsky, A simplified FEM eigenstrain residual stress reconstruction for surface treatments in arbitrary 3D geometries, Int. J. Mech. Sci. 138139 (2018) 457-466. doi:10.1016/j.ijmecsci.2018.02.016.

[12] E. Salvati, A.J.G. Lunt, S. Ying, T. Sui, H.J. Zhang, C. Heason, G. Baxter, A.M. Korsunsky, Eigenstrain reconstruction of residual strains in an additively manufactured and shot peened nickel superalloy compressor blade, Comput. Methods Appl. Mech. Eng. 320 (2017) 335-351. doi:10.1016/j.cma.2017.03.005.

[13] J. Everaerts, E. Salvati, F. Uzun, L. Romano Brandt, H. Zhang, A.M. Korsunsky, Separating macro- (Type I) and micro- (Type II+III) residual stresses by ring-core FIBDIC milling and eigenstrain modelling of a plastically bent titanium alloy bar, Acta Mater. 156 (2018) 43-51. doi:10.1016/J.ACTAMAT.2018.06.035.

[14] A.T. DeWald, M.R. Hill, Multi-Axial Contour Method for Mapping Residual Stresses in Continuously Processed Bodies, Exp. Mech. 46 (2006) 473-490. doi:10.1007/s11340-006-8446-5.

[15] F. Uzun, A.M. Korsunsky, On the identification of eigenstrain sources of welding residual stress in bead-on-plate inconel 740H specimens, Int. J. Mech. Sci. 145 (2018) 231-245. doi:doi.org/10.1016/j.ijmecsci.2018.07.007.

[16] S.J. Russell, P. Norvig, Artificial Intelligence: A Modern Approach, Prentice Hall, Englewood Cliffs, New Jersey, 1995.

[17] R. Quiza, O. López-armas, J.P. Davim, S.F. Wiesbaden, Hybrid Modeling and Optimization of Manufacturing, 2012. 
[18] K. Abdel-Tawab, A.K. Noor, Uncertainty analysis of welding residual stress fields, Comput. Methods Appl. Mech. Eng. 179 (1999) 327-344. doi:10.1016/S00457825(99)00045-6.

[19] U.O. Akpan, T.S. Koko, I.R. Orisamolu, B.K. Gallant, Practical fuzzy finite element analysis of structures, Finite Elem. Anal. Des. 38 (2001) 93-111. doi:10.1016/S0168874X(01)00052-X.

[20] M.-G. Na, J.-W. Kim, D.-H. Lim, Prediction of Residual Stress for Dissimilar Metals Welding At Nuclear Power Plants Using Fuzzy Neural Network Models, Nucl. Eng. Technol. 39 (2007) 337-348. doi:10.5516/NET.2007.39.4.337.

[21] M.C. Smith, A.C. Smith, R. Wimpory, C. Ohms, A review of the NeT Task Group 1 residual stress measurement and analysis round robin on a single weld bead-on-plate specimen, Int. J. Press. Vessel. Pip. 120-121 (2014) 93-140. doi:10.1016/j.ijpvp.2014.05.002.

[22] Special Metals Corporation, Inconel Alloy 740H, (2017) 24. http://www.specialmetals.com/assets/smc/documents/alloys/inconel/inconel-alloy-740h.pdf (accessed November 21, 2018).

[23] J. Ahn, E. He, L. Chen, R.C. Wimpory, J.P. Dear, C.M. Davies, Prediction and measurement of residual stresses and distortions in fibre laser welded Ti-6Al-4V considering phase transformation, Mater. Des. 115 (2017) 441-457. doi:10.1016/j.matdes.2016.11.078.

[24] T. Mura, Micromechanics of Defects in Solids, Netherlands: Kluwer Academic Publishers, Dordrecht, 1982.

[25] A.M. Korsunsky, Power Law Multi-Scaling of Material Strength, (2005) 1-14. http://arxiv.org/abs/cond-mat/0508653.

[26] Y.C. Toklu, F. Uzun, Analysis of Tensegric Structures by Total Potential Optimization 
Using Metaheuristic Algorithms, J. Aerosp. Eng. 29 (2016) 4016023. doi:10.1061/(asce)as.1943-5525.0000571.

[27] F. Uzun, Form-finding and analysis of an alternative tensegrity dome configuration, Adv. Struct. Eng. 20 (2017) 1644-1657. doi:10.1177/1369433216689570.

[28] F. Uzun, Form-finding of free-form tensegrity structures by genetic algorithm-based total potential energy minimization, Adv. Struct. Eng. 20 (2017) 784-796. doi:10.1177/1369433216664739.

[29] M. Mitchell, An Introduction to Genetic Algorithms, The MIT Press, Cambridge, Massachusetts, 1998.

[30] B.D. Conduit, N.G. Jones, H.J. Stone, G.J. Conduit, Design of a nickel-base superalloy using a neural network, Mater. Des. 131 (2017) 358-365. doi:10.1016/j.matdes.2017.06.007.

[31] F. Uzun, A.N. Bilge, The effect of carbon content and submerged arc welding process on hardness of carbon steels, J. Found. Appl. Phys. 4 (2017) 1-7.

[32] F. Uzun, A.N. Bilge, A Comprehensive Model for Hardness Evaluation of Low-Alloy Steels Based on Carbon Content and Ultrasonic Wave Velocity, Mater. Eval. 75 (2017) $1489-1496$.

[33] M.C. Smith, A.C. Smith, NeT bead-on-plate round robin: Comparison of transient thermal predictions and measurements, Int. J. Press. Vessel. Pip. 86 (2009) 96-109. doi:10.1016/j.ijpvp.2008.11.016. 